\title{
Conformación de un modelo pedagógico basado en el uso de Objetos de Aprendizaje aplicado a los procesos de enseñanza en la Universidad de Guadalajara
}

\author{
Saúl Gutiérrez Medina y Luis Alberto Gutiérrez Díaz de León \\ Universidad de Guadalajara, Av. Juárez No. 976, Col. Centro, C.P. 44100, Guadalajara, Jalisco, México. \\ saul.gutierrez@cuaad.udg.mx, luis.gutierrez@redudg.udg.mx
}

Fecha de recepción: 6 de noviembre 2015

Fecha de aceptación: 16 de diciembre 2015

\begin{abstract}
Resumen. La utilidad didáctica de los Objetos de Aprendizaje (OAs) en ambientes educativos mediados por tecnologías se enfatiza tras la revisión de distintos estudios que hablan de resultados satisfactorios en los aprendizajes mediante el empleo de OAs. La presente investigación aborda el caso de la asignatura de Arqueología de la Licenciatura en Turismo en la Universidad de Guadalajara, donde la necesidad de reestructurar los contenidos planteados por el uso aleatorio y no organizado de materiales didácticos tecnológicamente limitados, se tradujo en la adopción de un modelo sistemático para apoyar a sus docentes en los procesos de enseñanza y aprendizaje. La solución propuesta se enfoca en la creación de una colección de OAs, diseñados instruccionalmente con base en modelos pedagógicos sistemáticos. La evaluación de dos prototipos busca validar su contribución en favor del aprendizaje de los sitios estudiados para implantar su manejo en la asignatura como herramientas sustentadas pedagógicamente.
\end{abstract}

Palabras Clave: Objetos de Aprendizaje, Arqueología, Tecnología Educacional, Turismo, Educación, Pedagogía, Multimedia.

\section{Introducción}

El uso de Objetos de Aprendizaje se ha extendido de manera significativa en diferentes áreas del conocimiento durante los últimos años. Cada vez son más las investigaciones que ratifican sus aportes y utilidad en los ambientes de aprendizaje.

En un estudio reciente sobre OAs, [1] afirman que, dentro del contexto del aprendizaje en ambientes mediados por las TICs, las instituciones universitarias involucran innovaciones en los procedimientos donde la enseñanza se debe planear, ejercer y mediar con la aplicación de la virtualidad y de la interacción para la generación de un autoaprendizaje multidimensionado, secuencial y estructurado para que los alumnos entiendan su significatividad y aplicabilidad.

La Universidad de Guadalajara se encuentra inmersa en esta transformación sociológica, con un papel activo al ser formadora de profesionistas con habilidades en el manejo de las tecnologías y en los diferentes ámbitos que ésta abarca.

\subsection{Delimitación del problema}

La asignatura de Arqueología de la Licenciatura en Turismo del CUCEA es impartida con el uso de algunos elementos digitales aislados y no organizados de origen externo, adaptando su utilidad a los requerimientos de un contenido curricular en transformación, por lo que se busca apoyar su proceso de enseñanza mediante una colección de Objetos de Aprendizaje (OAs), cuyos contenidos se enfocarán en la descripción de las principales zonas arqueológicas mexicanas y en la gestión de las vías, servicios e información para la promoción de visitas turísticas a las mismas.

Se pretende que el Licenciado en Turismo, más que ser un experto en detalles históricos y técnicos sobre arqueología, sea un gestor profesional capaz de orientar y motivar a los turistas para visitar los sitios arqueológicos estudiados.

\section{Estado del Arte}

La educación es concebida como un sistema, donde el conocimiento es la materia prima que procesa el estudiante, el mecanismo de entrada lo constituye un instructor a través de un medio o ambiente instruccional, el 
proceso lo constituyen las actividades de aprendizaje interactivas cuyo resultado final será el aprendizaje obtenido por el estudiante; este es el proceso instruccional al que [2] refieren como un modelo sistemático donde los componentes como parte de un todo, se interrelacionan para trabajar conjuntamente hacia una misma meta: un aprendizaje exitoso.

Destaca lo que afirma [3] sobre el uso de las tecnologías y sus beneficios en los entornos educativos, al señalar que "La capacidad de los sistemas informáticos multimedia para mostrar las interacciones dinámicas de los componentes sugiere que esta tecnología tiene el potencial para ayudar a los estudiantes a desarrollar modelos que representen el mundo de una manera precisa y accesible" (p. 95).

Tal es el caso de los OAs, al verificar varios estudios referentes a la producción de entidades digitales aplicadas en distintos contextos de la educación, basados en metodologías sistematizadas para la estructuración y la evaluación del OA, considerando aspectos técnicos y pedagógicos en todos los casos; su utilización se ha extendido como nuevo paradigma en el diseño de este tipo de materiales, según [4] de la Universidad Central de Venezuela, al destacar las bondades de estos objetos tras un estudio sobre su aplicación en la enseñanza de sistemas de bases de datos.

De la misma forma lo refieren [1] de la Universidad Autónoma de Tamaulipas, en su estudio sobre la construcción y transferencia del conocimiento mediante el uso de OAs, en el que produjeron un ejemplar tras un análisis documental sobre cómo abordar problemáticas de la educación a distancia dentro del contexto y características de los alumnos en ambientes mediados por las TICs; de igual forma, sus resultados reportan una buena adecuación de la interfaz y de los recursos multimedia para el aprendizaje del módulo desarrollado, donde la evaluación pedagógica arrojó un alto nivel de aprobación por la mayoría de los alumnos que utilizaron el OA. Los investigadores [5] de la Universidad Austral de Chile, desarrollaron un modelo colaborativo de construcción de OAs enfocado pedagógicamente en áreas de la salud.

Por su parte, [6] de la Universidad Politécnica de Aguascalientes, produjeron un OA para el área de ingeniería en sistemas con fundamentos pedagógicos y metodológicos basado en mapas mentales; en su proceso contempla indicadores técnicos y pedagógicos que los alumnos meta calificaron, llegándose a la conclusión de que el OA es una herramienta eficaz de apoyo para la enseñanza y aprendizaje.

\section{Metodología}

El presente proyecto se basa en el modelo ADDIE (Análisis, Diseño, Desarrollo, Implementación y Evaluación) para su realización. Este considera la relación entre el estudiante, el instructor y los materiales, dentro los entornos tecnológicos educativos. Se trata de un flujo de procesos lineal pero dinámico, que conforma un modelo de inter-relaciones sistemático simple y genérico; esto le concede la facilidad de adecuarse a cualquier entorno de aprendizaje al ofrecer una guía flexible para el diseño y producción de materiales educativos, que puede ser modificado y adaptado de acuerdo con el proyecto instruccional que se requiera desarrollar. Dentro de sus tareas considera el desarrollo de evaluaciones formativas y sumativas, fase a la que adaptamos el modelo diseñado por [2], también basado en la teoría de sistemas, en función del tamaño del grupo meta y la consistencia de las recomendaciones de estos autores para su aplicación; éste será incluido dentro de la metodología considerando su valor y enfoque pedagógico orientado al diseño instruccional, con fundamentos teórico-pedagógicos reconocidos.

De este modelo se extraen las evaluaciones mediante instrumentos que consideran escalas tipo Likert, que van de 1 a 5 puntos, desde la valoración de "En total desacuerdo" con valor de 1 punto, hasta "Totalmente de acuerdo" con un valor de 5 puntos, aplicadas a un experto en diseño para valorar los elementos técnicos, la evaluación por un experto en contenido para valorar elementos pedagógicos, además de la evaluación de grupo pequeño, donde la encuesta es aplicada por los alumnos del grupo meta, con indicadores de los dos anteriores para valorar ambos aspectos .

Los indicadores se han elegido mediante una adecuación basada en las dimensiones del modelo LORI (Learning Object Review Instrument), por su especial enfoque en la evaluación de OAs, de las que se han considerado:

a) Calidad de los contenidos

b) Adecuación de los objetivos de aprendizaje

c) Retroalimentación y adaptabilidad

d) Motivación

e) Diseño y presentación

f) Usabilidad

g) Adecuación a estándares 
Las evaluaciones consisten en escalas de Likert, con 11 indicadores para el experto en diseño; 10 indicadores para el experto en contenido; y la evaluación de grupo pequeño, con 15 indicadores. Los indicadores se han adaptado a las dimensiones del modelo LORI mencionadas, por lo que pueden encontrarse uno, dos, tres o más que midan aspectos específicos pertenecientes a una misma dimensión; por ejemplo, la Calidad de los contenidos, es una dimensión que se alimentará de los resultados de tres indicadores en la encuesta identificados con los números 2,8 y 15 en este caso:

2. El vocabulario utilizado en el OA es comprensible;

8. Las instrucciones son claras y precisas;

15. La redacción es clara y entendible para una lectura amena.

Éstos indicadores analizan aspectos que tienen que ver con la calidad de los contenidos y se agrupan en ésta dimensión del modelo LORI para mejor comprensión.

\section{Diseño Instruccional}

\subsection{Análisis}

El grupo meta comprende estudiantes de la asignatura de Arqueología en su mayoría del sexo femenino en un rango de edad de los 18 a los 33 años, con promedio de 22 años en más de la mitad del grupo; con nivel académico general de preparatoria, que serán enfocados hacia la gestión y promoción de emplazamientos arqueológicos. El período en que se aplicaron los pilotajes a los grupos meta fue el 2010-B.

Se estableció también que los contenidos de los OAs deberán ofrecer un video introductorio. Finalmente, en función de su importancia turística y cultural en nuestro país y el contexto local, se define que los sitios arqueológicos elegidos para la fase de los pilotajes serían Uxmal y Guachimontones (dos prototipos), incluyendo para éste último la generación del video con recursos propios de la Universidad de Guadalajara.

\subsection{Diseño}

Se planteó la creación de OAs visualmente equilibrados y llamativos, basados en los criterios básicos de usabilidad; estos incluirán actividades que los relacionen y conduzcan directamente con el objetivo instruccional.

Tanto los contenidos instruccionales como su estructuración en los OAs fueron definidos por el experto en contenido (docente de la asignatura de Arqueología) delimitando secciones por módulos (Fig. 1).

Se definieron cinco módulos para ambos OAs, accesibles mediante una barra horizontal de botones para organizar la información de forma lógica y secuencial, para una mayor usabilidad al facilitar la navegación a través de la organización de los contenidos. Estos módulos son:

1. Inicio. Este contiene el Objetivo instruccional y el video introductorio.

2. El Sitio. Contiene información propia del sitio arqueológico.

3. Servicios Turísticos. Contiene información sobre servicios de transporte, hospedaje, alimentación e información turística del sitio.

4. Actividad. Contiene las instrucciones para la actividad de aprendizaje interactiva que realizarán los alumnos de forma externa.

5. Evaluación. Contiene la evaluación para medir los aprendizajes de la información contenida en el OA.

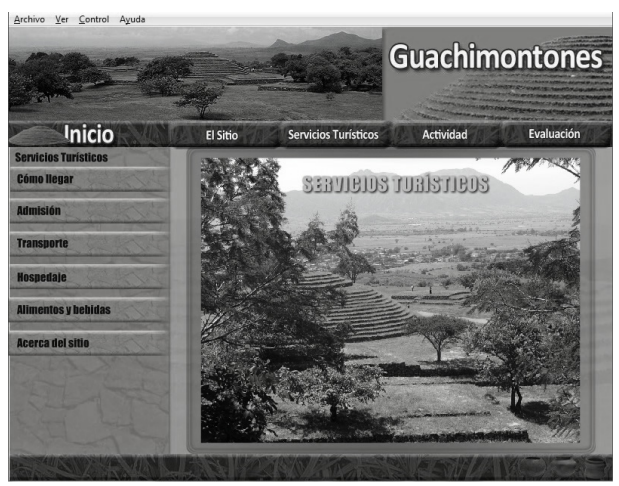

Figura 1. Vista de la interfaz gráfica con la estructura definida para ambos prototipos 


\subsection{Desarrollo}

Para el desarrollo de los OAs se utilizaron diferentes herramientas multimedia, básicamente Adobe Photoshop CS4 y CS5 para generar los gráficos para los OAs, Adobe Flash CS4 y CS5 para integrar y generar los OAs, Adobe Premiere CS5 para editar videos; así mismo, equipo de grabación de audio profesional, software para edición de audio Sony Vegas y Audacity; cámara profesional Panasonic para grabación de video y cámara fotográfica; equipos de cómputo mac y pc.

\subsection{Implantación}

El desarrollo de los OAs generó varios archivos para su ejecución; el principal es el .swf que una vez puesto en marcha llama a otros elementos fuera de este que lo integran, por lo que se empaquetaron mediante la compresión en formato rar, que ofrece una mejor compresión por ahorro de espacio de almacenamiento en relación al zip, además de permitir la recuperación física de datos dañados. Dicha compresión genera un archivo único con todos los componentes del OA, facilitando subirse y descargarse rápidamente para su distribución, en este caso a través de Drop Box para realizar los pilotajes, desde los expertos en diseño y contenido hasta los grupos meta.

\subsection{Evaluación}

El grupo meta que realizó la evaluación de Uxmal, muestra una tendencia que promedia en $90 \%$ su nivel de aceptación del OA en general. La mayoría de las valoraciones para los 15 indicadores se encuentran entre los puntos 4 y 5, es decir, entre De Acuerdo y Totalmente de Acuerdo, esto es un gran nivel de aprobación. (Fig. 2)

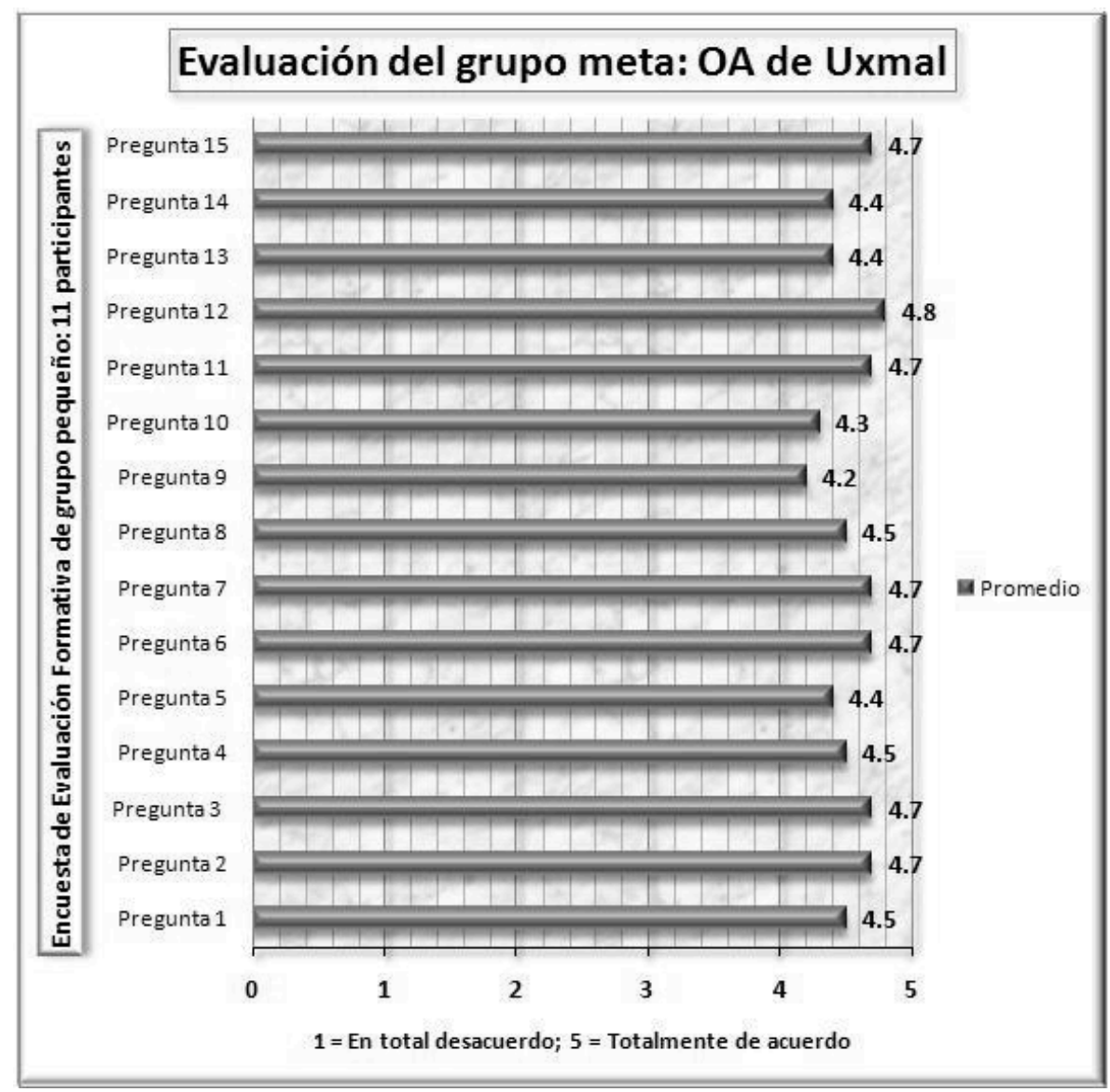

Figura 2. Evaluación del OA de Uxmal por el grupo meta 
Para el grupo que valoró el OA de Guachimontones, los resultados muestran una tendencia semejante. Los promedios en todos los indicadores están por arriba de los 4 puntos, en varios casos en los 5 puntos. En este caso la tendencia en el promedio de aceptación general de este OA por parte del grupo meta asciende al $93 \%$. (Fig. 3)

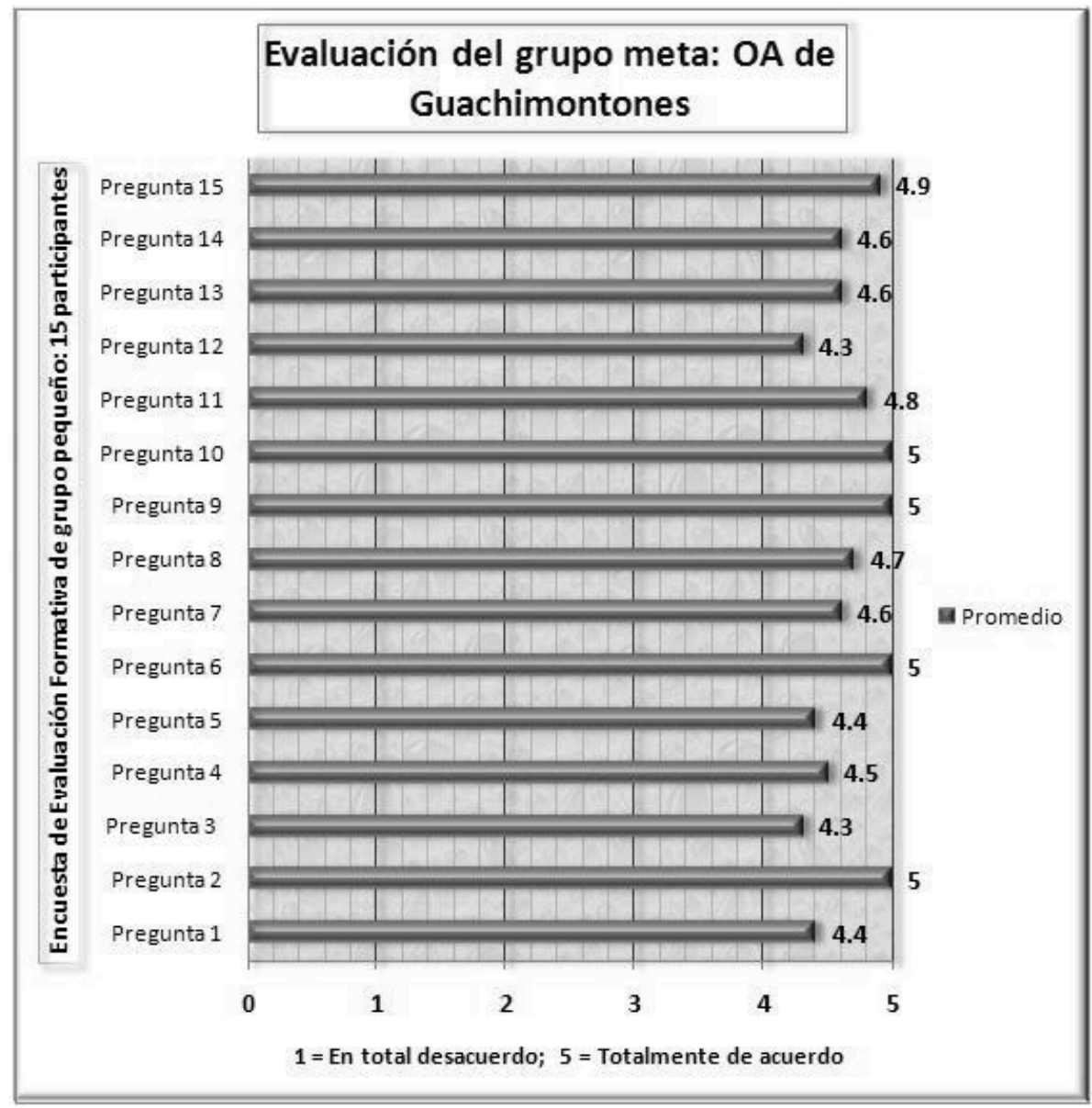

Figura 3. Evaluación del OA de Guachimontones por el grupo meta

\section{Evaluación de expertos}

\subsection{Evaluación del Experto en Diseño}

De acuerdo al modelo LORI, las dimensiones valoradas en la evaluación fueron:

- Adecuación de los objetivos de aprendizaje

- Diseño y presentación

- Usabilidad

- Adecuación a estándares internacionales (metadatos)

El promedio con que fueron calificados ambos OAs incluyendo las cuatro dimensiones mencionadas es de 3.9 con base en la escala de Likert, lo que significa un nivel aprobatorio muy aceptable por parte del evaluador.

\subsection{Evaluación del Experto en Contenido}

En este instrumento se consideraron las siguientes dimensiones del modelo LORI:

- Calidad de los contenidos

- Adecuación de los objetivos de aprendizaje

- Retroalimentación y adaptabilidad 
La valoración promedio general para ambos OAs incluyendo las tres dimensiones mencionadas fue de 4.8, lo que se traduce que fueron aceptados o aprobados en un $96 \%$ por parte del experto en contenido, un grado muy significativo que confirma que se ha alcanzado el objetivo perseguido a lo largo del presente trabajo, aun cuando existen detalles que se pueden mejorar.

\section{Conclusiones}

Basados en los resultados y tras los análisis realizados, los OAs piloteados obtuvieron una gran aceptación por parte de los estudiantes de ambos grupos meta, mostrando promedios importantes en las 6 dimensiones del modelo LORI valoradas.

Al ser sometidos a metodologías de construcción sistemáticas, los OAs son elementos que favorecen y motivan a los alumnos hacia los aprendizajes, acorde con los resultados de este estudio, por lo que su utilidad en apoyo a los docentes y los procesos de enseñanza, queda demostrada una vez más también en este proyecto de investigación.

Dentro de las observaciones y recomendaciones realizadas por los expertos, se pondrá especial atención en las áreas de usabilidad y diseño, con la finalidad de pulir los OAs de tal forma que incremente la viabilidad de llevarlos a su aplicación en el marco del contexto real.

Entre éstas mejoras se consideran las adecuaciones en los tamaños de las fuentes y su contrastación de color con los fondos para permitir un mayor grado de legibilidad; también se agregarán más imágenes en secciones que carecen de ellas, incluyendo la imagen estática de un mapa de ubicación de la zona en la sección de Ubicación/Localización.

El botón del Mapa del $O A$ se reubicará hacia una sección mayormente visible, probablemente al costado derecho del botón de inicio, para facilitar al usuario la búsqueda y ubicación de cualquier sección en el OA, además de agregar links dentro de todos los elementos mostrados para incrementar la navegabilidad y usabilidad de la herramienta.

Cabe destacar la opinión del experto en contenido en torno a la estructuración de los contenidos, su distribución y su relación con la información expuesta en los videos, en el sentido de haber dado respuesta cabal a lo esperado de estos OAs, con especial énfasis en lo apropiado de la actividad de aprendizaje y su adecuación para el alcance del objetivo instruccional establecido.

De lo anterior se deduce que se han alcanzado los objetivos del presente proyecto; se modelaron y pilotearon dos OAs tras una acertada definición de su diseño y estructura con base en metodologías, estándares y modelos instruccionales sistematizados; se efectuaron las evaluaciones formativas con resultados que dan cuenta del alcance y utilidad didáctica de ambos OAs, con serias posibilidades de proyectarse en el fomento del uso de las TICs como medios para la estimulación de aprendizajes, una vez que sean aplicadas las recomendaciones emitidas por los expertos para su empleo en el contexto real.

Finalmente, con la prueba fehaciente ofrecida por estos resultados al comprobar el aporte pedagógico de los OAs diseñados, los trabajos futuros podrán orientarse no sólo a completar la colección de OAs contemplados por la asignatura a la cual se enfocan, sino también a la integración de nuevas zonas arqueológicas que sean descubiertas y a su estudio clasificado por regiones, países o culturas, ya que su contribución posibilita ampliar el panorama para su inserción en entornos virtuales de aprendizaje. En ese tenor, se sugieren investigaciones en diseño instruccional que abarquen un espectro completo dentro de las modalidades educativas no convencionales y a distancia, de tal forma que se modelen cursos en línea de arqueología totalmente constructivistas y autogestivos, aplicables en cualquier ambiente de instrucción que requiera del dominio de estos conocimientos mediante el uso de colecciones de OAs adecuados a las necesidades pedagógicas y docentes para cada caso en particular.

Para tal fin, se sugiere también realizar investigaciones que coadyuven a actualizar y enriquecer funcional y pedagógicamente la estructura de los OAs de acuerdo a los avances tecnológicos aplicables, mediante el uso de desarrollos o recursos gratuitos disponibles en la red tales como mapas y videos interactivos, visitas a museos y recorridos virtuales, etc., algunos ya disponibles, otros en proceso, lo cual incluye el uso de herramientas para su desarrollo como el lenguaje HTML5 que ofrece funcionalidades que se presume desplazarán aplicativos como el Adobe Flash y para los que ya existen utilerías de conversión de éste último al primero (Google Swiffy).

Todas estas sugerencias se enfocan a ofrecer experiencias más reales y completas que faciliten al estudiante una aprehensión más exacta y más significativa de los conocimientos, además de dotar a los docentes de herramientas que apoyen sus esfuerzos en la transferencia de esos conocimientos mediante el uso de las nuevas tecnologías de información y comunicación. 


\section{Referencias}

1. Hernández, M., y Padilla, G. E. Los objetos de aprendizaje, construcción y transferencia del conocimiento: la planeación en la UNAED para un entorno virtual. En Muñoz, A. J., Álvarez, R. F. J. \& Ochoa, X. (Eds.), Tercera conferencia Latinoamericana de tecnología de objetos de aprendizaje LACLO 2008. (pp. 197-203). Aguascalientes, Ags., México: Universidad Autónoma de Aguascalientes (2008).

2. Dick, W., Carey L., y Carey, J. The systematic design of instruction. ( $5^{\text {th }}$ ed). New York: Allyn \& Bacon (2001).

3. Winn, W. Cognitive perspectives in psychology. (D. H. Jonassen, Ed.) Cognitive Psychology, (2), 79-112. Simon and Schuster Macmillan. Recuperado el 27 de octubre de 2011 de http://aect.org/edtech/04.pdf (1996).

4. Hernández, Y. C., y Silva, A. Experiencia en la construcción de un objeto de aprendizaje para apoyar el proceso de enseñanza y aprendizaje de los sistemas de bases de datos orientados a objetos. En Muñoz, A. J., Álvarez, R. F. J. \& Ochoa, X. (Eds.), Tercera conferencia Latinoamericana de tecnología de objetos de aprendizaje LACLO 2008. (pp. 139-146). Aguascalientes, Ags., México: Universidad Autónoma de Aguascalientes (2008).

5. Bucarey, S., Vieira, A., Cabezas, X., Carrapatoso, E., y Vaz, C. Descripción de pasos básicos para la construcción colaborativa de objetos de aprendizaje y uso del repositorio médico compartido MELOR. En Muñoz, A. J., Álvarez, R. F. J. \& Ochoa, X. (Eds.), Tercera conferencia Latinoamericana de tecnología de objetos de aprendizaje LACLO 2008. (pp. 5561). Aguascalientes, Ags, México: Universidad Autónoma de Aguascalientes (2008).

6. Juárez, O. y Reyes, P. Objetos de Aprendizaje como apoyo a la educación y fomento al aprendizaje didáctico. En Muñoz, A. J., Álvarez, R. F. J. \& Ochoa, X. (Eds.), Tercera conferencia Latinoamericana de tecnología de objetos de aprendizaje LACLO 2008. (pp. 25-32). Aguascalientes, Ags., México: Universidad Autónoma de Aguascalientes (2008). 